\section{Revista de CIENCIAS AMBIENTALES Tropical Journal of Environmental Sciences}

Revista de Ciencias Ambientales (Trop J Environ Sci)

e-ISSN: 2215-3896

(Julio-Diciembre, 2019). Vol 53(2): 111-131

DOI: https://doi.org/10.15359/rca.53-2.5

Open Access: www.revistas.una.ac.cr/ambientales e-mail: revista.ambientales@una.ac.cr

Fonseca-González W., Villalobos-Chacón R., Rojas-Vargas M.

\title{
Potencial de mitigación del cambio climático de los ecosistemas forestales caducifolios en Costa Rica: modelos predictivos de biomasa y carbono
}

\author{
Potential Mitigation of Climate Change of Deciduous Forest Ecosystems in Costa \\ Rica: Predictive Models of Biomass and Carbon
}

\author{
William Fonseca-González ${ }^{a}$, Ronny Villalobos-Chacón ${ }^{b}$, Marilyn Rojas-Vargas $^{c}$
}

[Recibido: 29 de marzo 2019, Aceptado: 6 de mayo 2019, Corregido: 27 de mayo 2019, Publicado: 1 de julio 2019]

\section{Resumen}

La cobertura vegetal, principalmente los bosques, cumple un papel fundamental en la mitigación del cambio climático. Se calculó la biomasa y el carbono almacenado para bosques caducifolios, primarios y secundarios, en la región del Pacífico Norte de Costa Rica y se desarrollaron sus modelos predictivos correspondientes. Con un muestreo aleatorio por medio de parcelas anidadas y a través del método destructivo, se evaluó la cantidad de biomasa arbórea y subterránea de árboles de diámetro promedio, la necromasa y la vegetación herbácea. Los modelos se construyeron para el árbol completo y sus componentes (tronco, raíz, ramas y hojas), usando como variable predictora el diámetro normal, en los modelos para cuantificar la biomasa y el carbono por hectárea, se utilizó el área basal. Todos ellos presentaron ajustes muy satisfactorios $\left(\mathrm{R}^{2}>0.93\right)$ y predijeron tanto la biomasa como el carbono con bajos errores de estimación. La biomasa total en el ecosistema fue de $140.0 \mathrm{Mg} \mathrm{ha}^{-1}\left(65.5 \mathrm{Mg} \mathrm{C} \mathrm{ha}^{-1}\right)$, un 93.6 $\%$ corresponde a biomasa o carbono en los árboles. La fracción de carbono entre los componentes varió entre 39.1 y $42.8 \%$. El factor de expansión de biomasa para incluir hojas, rama y raíz fue de $2.09 ; 1.69$, hojas y ramas, y 1.37 , solo para la raíz. Los modelos elegidos se caracterizan por su fácil aplicación, por usar como variable predictora el diámetro o el área basal, ambos fáciles de medir o de calcular en todo inventario forestal. Asimismo, la biomasa y el carbono calculados en el nivel de ecosistema y por árbol, los valores de fracción de carbono en la biomasa y los factores de expansión están entre los rangos citados en otras investigaciones, convirtiéndolos, así, en indicadores nacionales que facilitan estimar, con mayor exactitud, el aporte del ecosistema a la mitigación del cambio climático.

Palabras clave: alometría; bosque tropical; inventario forestal; servicios ambientales

\section{Abstract}

Plant cover, mainly forests, plays a key role in mitigating climate change. Biomass and stored carbon were calculated for primary and secondary deciduous forests in the Northern Pacific region of Costa Rica, and predictive models for biomass and carbon were developed. Using random sampling of nested plots and the destructive method, the amount of arboreal and subterranean biomass of trees of average diameter, necromass and herbaceous vegetation were evaluated. The models were constructed for the entire tree and its components (trunk, roots, branches and leaves), using normal diameter as a predictor variable; in models to quantify biomass and carbon per hectare, basal

a Investigador y académico de la Escuela de Ciencias Ambientales e Instituto de Investigación y Servicios Ambientales, Universidad Nacional, Costa Rica, wfonseca@una.ac.cr, https://orcid.org/0000-0002-4546-9035

b Ingeniero forestal, investigador y académico de la Escuela de Ciencias Ambientales, Universidad Nacional, Costa Rica, ronny.villalobos. chacon@una.ac.cr, https://orcid.org/0000-0003-2606-5286

c Ingeniera forestal, investigadora y académica de la Escuela de Ciencias Ambientales, Universidad Nacional, Costa Rica, marilyn.rojas. vargas@una.ac.cr, https://orcid.org/0000-0001-7051-8399

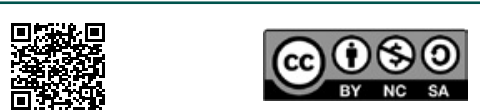




\section{Revista de CIENCIAS AMBIENTALES Tropical Journal of Environmental Sciences}

Revista de Ciencias Ambientales (Trop J Environ Sci)

e-ISSN: 2215-3896

(Julio-Diciembre, 2019). Vol 53(2): 111-131

DOI: https://doi.org/10.15359/rca.53-2.5

Open Access: www.revistas.una.ac.cr/ambientales e-mail: revista.ambientales@una.ac.cr

Fonseca-González W., Villalobos-Chacón R., Rojas-Vargas M.

area was used. All models fit the data well $\left(\mathrm{R}^{2}>0.93\right)$ and predict biomass and carbon with low estimation errors. The total biomass in the ecosystem was $140.0 \mathrm{Mg} \mathrm{ha}^{-1}\left(65.5 \mathrm{Mg} \mathrm{C} \mathrm{ha}^{-1}\right)$, with $93.6 \%$ corresponding to biomass or carbon in trees. The carbon fraction among the components varied between $39.1 \%$ and $42.8 \%$, while the biomass expansion factor to include leaves, branches and roots was 2.09; 1.69 to include leaves and branches, and 1.37 for roots alone. All the models chosen to estimate biomass or carbon are characterized by their ease of application, using either diameter or basal area as a predictor variable, both of which are simple to measure or calculate in any forest inventory. Biomass and carbon calculated at the ecosystem and tree levels, biomass carbon fraction values and expansion factors are within the ranges cited in other investigations, thus making them national indicators that facilitate more accurate estimations of the contribution of ecosystems to the mitigation of climate change.

Keywords: allometry; environmental services; forest inventory; tropical forest

\section{Introducción}

El cambio climático producto de las actividades antrópicas - consumo de combustibles fósiles, deforestación, desarrollo de la agricultura y de la ganadería, entre otras - ha sido debatido y reconocido en el nivel mundial (Intergovernmental Panel of Climate Change [IPCC], 2013), igualmente, se reconoce la importancia de los bosques como mitigadores de tal cambio, al absorber el dióxido de carbono $\left(\mathrm{CO}_{2}\right)$ atmosférico a través de la fotosíntesis, para acumular carbono en su biomasa (Le Quéré et al., 2012).

Los ecosistemas de la zona de vida Bosque Seco Tropical ocupan casi la mitad del trópico y se encuentran muy influenciados por aspectos socioeconómicos, lo que ha producido alta degradación y amenazas por sobreexplotación e incendios recurrentes (Rodríguez, Armenteras y Morales, 2004). El remanente se presenta como parches aislados dentro de un paisaje dominado por actividades agropecuarias (Álvarez et al., 2012), haciéndolos muy sensibles a los efectos del cambio climático, modificando las tasas de crecimiento, aumentando la mortalidad de los árboles, incrementando la frecuencia de las perturbaciones (Álvarez et al., 2012; Miles et al., 2006; Phillips et al., 2009, 2010). Esto motiva a adelantar acciones de restauración, mediante la recuperación de áreas degradadas y el manejo de vegetación secundaria (Vieira y Scariot, 2011), ya que la tendencia de transformación del bosque seco, globalmente, continúa (Miles et al., 2006) y es el uso agropecuario la principal causa de reducción del área, así como de la fragmentación.

El bosque seco tropical ocupa el penúltimo lugar en extensión, mundialmente, el tercero con mayor aporte de biomasa y el cuarto en cantidad de carbono depositado (Houghton y Skole, 1990). Este ecosistema, a pesar de su extensión y de su aporte a la mitigación del cambio climático, ha sido poco estudiado (Álvarez et al., 2012). De aquí surgió la investigación cuyo objetivo primordial fue cuantificar el carbono almacenado y desarrollar modelos predictivos tanto de biomasa como de carbono, información requerida para compensar a los propietarios de bosque por el carbono almacenado, a través del programa de Pago por Servicios Ambientales, operado por el Fondo Nacional de Financiamiento Forestal de Costa Rica (FONAFIFO). Adicionalmente, se

(cc)




\section{Revista de CIENCIAS AMBIENTALES Tropical Journal of Environmental Sciences}

Revista de Ciencias Ambientales (Trop J Environ Sci) e-ISSN: 2215-3896

(Julio-Diciembre, 2019). Vol 53(2): 111-131 DOI: https://doi.org/10.15359/rca.53-2.5

Open Access: www.revistas.una.ac.cr/ambientales e-mail: revista.ambientales@una.ac.cr Fonseca-González W., Villalobos-Chacón R., Rojas-Vargas M.

calcularon factores de expansión de biomasa, al igual que la fracción de carbono en los distintos componentes de la biomasa y la necromasa.

\section{Metodología}

\section{1 Área de estudio}

La zona de estudio comprende el pacífico seco de Costa Rica, abarcando los ecosistemas forestales caducifolios en las provincias Guanacaste y Puntarenas, caracterizado por presentar una variación térmica estacional fuerte, con un periodo seco de hasta 6 meses, precipitaciones entre 400 y $1500 \mathrm{~mm}$ por año, elevada luminosidad, poca capacidad homeostática, polinización anemófila y las semillas dispersadas por insectos. Su composición en estructura está definida por solo un estrato arbóreo de altura media, baja diversidad florística y dominancia de especies con ritmos fenológicos muy marcados.

\subsection{Diseño de muestreo, selección de sitios y distribución de las unidades de muestreo}

El diseño de muestreo fue aleatorio sin remplazo, se cubrió la zona con una malla cuadrada de $6 \mathrm{~km}$. La población fue de 350 cuadrículas y se muestreó a una intensidad del 4 \% (14 sitios), tomando en consideración lo complejo del estudio y la disponibilidad de recursos económicos (Figura 1).

En cada cuadrícula, se eligió 1 o más propiedades con cobertura boscosa de distintas edades, para obtener la mayor variabilidad en densidad de biomasa. Se establecieron, en promedio, 5 unidades de muestreo (81 en total) de distinto tamaño, según el componente de biomasa por evaluar.

\subsection{Evaluación de la biomasa}

El material leñoso vivo (árboles y arbustos) con Dap $\geq 10 \mathrm{~cm}$ (diámetro normal a $1.3 \mathrm{~m}$ sobre el nivel del suelo) se evaluó en parcelas rectangulares de $20 \times 25 \mathrm{~m}\left(500 \mathrm{~m}^{2}\right) \mathrm{y}$, en un cuadrante o subparcela de $125 \mathrm{~m}^{2}$, los árboles con Dap entre $2.5 \mathrm{~cm}$ y $9.9 \mathrm{~cm}$ (Figura 2). Para cada individuo, se identificó la especie. Posteriormente, se definieron 4 clases diamétricas: $<10$ $\mathrm{cm}, 10-19.9,20-39.9 \mathrm{y} \geq 40 \mathrm{~cm}$, y para cada rango diamétrico se determinó la especie de mayor índice de valor de importancia (IVI), 42 para la zona en estudio (Apéndice 1).

La biomasa de los árboles de las especies con mayor IVI y de Dap $\leq 40 \mathrm{~cm}$ se determinó haciendo uso del método destructivo, que consiste en derribar el árbol y pesar en el campo (peso húmedo en $\mathrm{kg}$ ) cada componente por separado (fuste, ramas, hojas y raíz). La raíz se extrajo con una retroexcavadora y pulif (tipo tecle manual que funciona de forma horizontal); posteriormente, se lavó para eliminar componentes como piedra y tierra. Se continuó con el proceso de pesado, aproximadamente, 2 horas después de secadas al aire. En los árboles de las clases diamétricas mayores a $40 \mathrm{~cm}$, se determinó el diámetro en pie, en secciones de $2 \mathrm{~m}$, con la ayuda

\begin{tabular}{|c|c|c|}
\hline 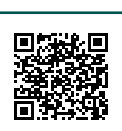 & (c) (i) @) (2) & 113 \\
\hline
\end{tabular}




\section{Revista de CIENCIAS AMBIENTALES Tropical Journal of Environmental Sciences}

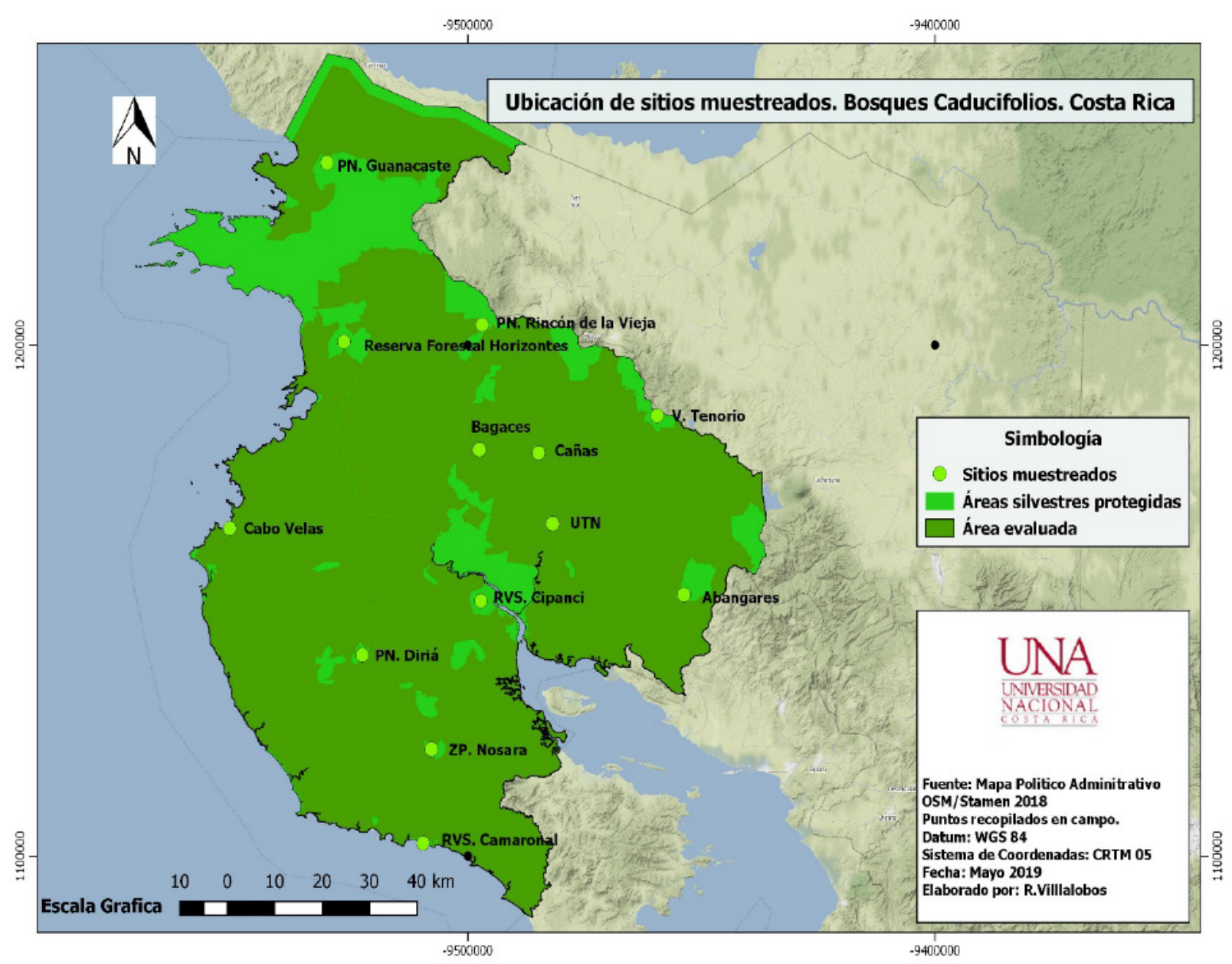

Figura 1. Sitios elegidos para establecer unidades de muestreo y evaluar la biomasa.

de un dendrómetro modelo CRITERION RD 1000, y el volumen se calculó con la fórmula de Smalian. La biomasa del fuste para cada individuo se cuantificó como el producto del volumen por la densidad específica de la madera (Chave et al., 2014; Cifuentes et al., 2015). La biomasa total del árbol (incluidas ramas, hojas y raíz) se obtuvo haciendo uso de factores de expansión de biomasa (Dixon, 1995; IPCC, 2006) calculados por especie para las clases inferiores a $40 \mathrm{~cm}$, en la misma investigación. La muestra para biomasa arbórea (fuste, ramas, raíz y hojas) fue de 141 árboles.

La vegetación herbácea y leñosa pequeña consistió en gramíneas, bejucos, helechos, plantas pequeñas y arbustos o regeneración de árboles con Dap $<2.5 \mathrm{~cm}$; se cuantificó en una parcela de 1 x $1 \mathrm{~m}$ ubicada en cada esquina de la parcela de $500 \mathrm{~m}^{2}$ (Figura 2). La necromasa fina

\begin{tabular}{|c|c|c|c|c|c|}
\hline 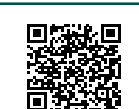 & (c) (i) (2) (2) & 8 & 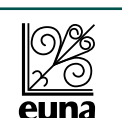 & 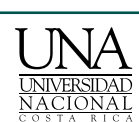 & 114 \\
\hline
\end{tabular}




\section{Revista de CIENCIAS AMBIENTALES Tropical Journal of Environmental Sciences}

Revista de Ciencias Ambientales (Trop J Environ Sci) e-ISSN: 2215-3896

(Julio-Diciembre, 2019). Vol 53(2): 111-131 DOI: https://doi.org/10.15359/rca.53-2.5

Open Access: www.revistas.una.ac.cr/ambientales e-mail: revista.ambientales@una.ac.cr Fonseca-González W., Villalobos-Chacón R., Rojas-Vargas M.

compuesta por material leñoso con diámetro $<2 \mathrm{~cm}$ y hojarasca se estimó en 4 parcelas de 0.5 x $0.5 \mathrm{~m}$, así como la necromasa gruesa (material leñoso con diámetro $\geq 2 \mathrm{~cm}$ ), en una parcela de 5 x $5 \mathrm{~m}$, ambas situadas aleatoriamente en la parcela de $500 \mathrm{~m}^{2}$ (Figura 2). Cada componente de la biomasa o la necromasa se pesó en húmedo en el campo y se tomó una muestra de aproximadamente $1 \mathrm{~kg}$, para determinar la materia seca (a $60{ }^{\circ} \mathrm{C}$ durante 72 horas) y la fracción de carbono en el laboratorio (Fonseca, Alice y Rey-Benayas, 2009).

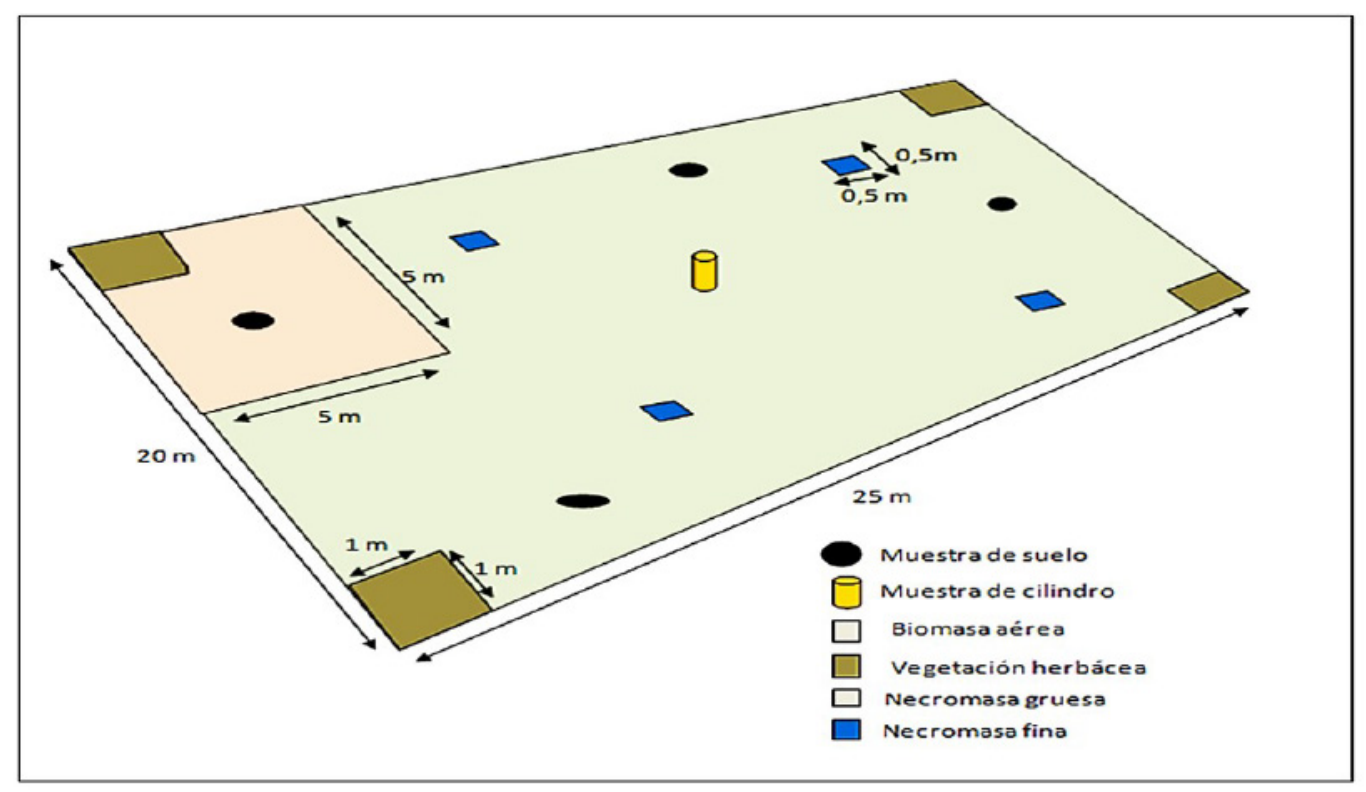

Figura 2. Unidad de muestreo para estimar biomasa y carbono.

\subsection{Cálculo del factor de expansión de biomasa (FEB)}

El factor de expansión se utiliza para calcular la biomasa de componentes como ramas, hojas y raíz, cuando se usa el método no destructivo. Es el cociente entre la biomasa aérea total y la biomasa del fuste, entre la biomasa del fuste y la biomasa de la raíz. La biomasa seca total del árbol se obtiene al multiplicar este cociente por el volumen del fuste y por la densidad específica de la madera (Segura, Kanninen, Alfaro y Campos, 2000; Segura y Kanninen, 2002).

\subsection{Determinación de la fracción de carbono en biomasa}

La muestra seca de cada componente de biomasa se trituró a 10 micras, para determinar la materia orgánica (MO). El análisis de esta última se realizó haciendo uso de la metodología propuesta por Walkey y Black (Álvarez y Marín, 2011). El tamaño de la muestra varió por

\begin{tabular}{|c|c|c|}
\hline 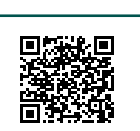 & (c) (1) () (2) & 115 \\
\hline
\end{tabular}




\section{Revista de CIENCIAS AMBIENTALES Tropical Journal of Environmental Sciences}

Revista de Ciencias Ambientales (Trop J Environ Sci) e-ISSN: 2215-3896

(Julio-Diciembre, 2019). Vol 53(2): 111-131 DOI: https://doi.org/10.15359/rca.53-2.5

Open Access: www.revistas.una.ac.cr/ambientales e-mail: revista.ambientales@una.ac.cr Fonseca-González W., Villalobos-Chacón R., Rojas-Vargas M.

componente, considerando la disponibilidad de recursos económicos, siempre que el error de estimación fuera inferior al $8 \%$.

\subsection{Ajuste de modelos}

Los modelos se ajustaron mediante el método de mínimos cuadrados ordinarios con el programa estadístico Statgrapihcs Centurion XVI. Se ensayaron aproximadamente 25 modelos para la biomasa y el carbono de cada componente del árbol (hojas, ramas, fuste y raíz), la biomasa leñosa del árbol (fuste, ramas y raíz) y el individuo total, usando como variable independiente el Dap. Un número similar de modelos se probó, con el fin de predecir la biomasa y el carbono por hectárea (modelos agregados), para el componente arbóreo (total de árboles/ha, incluyendo hojas, ramas, fuste y raíz), así como para el componente leñoso (ramas, raíz y fuste) y el nivel de ecosistema (biomasa del total de árboles, biomasa de la vegetación herbácea y la necromasa). En este caso, se utilizó el área basal ha $\mathrm{ha}^{-1}(\mathrm{G})$ como variable independiente.

El rango diamétrico de los modelos en el nivel de árbol osciló entre 4.1 y $80.2 \mathrm{~cm}$ y, para los modelos agregados, tuvo una amplitud en área basal de 5.0 a $52.5 \mathrm{~m}^{2}$. En ambos casos, el número de especies de mayor IVI fue 42. En la selección de la ecuación de mejor ajuste, se tomó como referencia la metodología expuesta por Salas (2002) y Segura y Andrade (2008), considerando los siguientes estadísticos: $\mathrm{R}$ (coeficiente de correlación), $\mathrm{R}^{2}$ (coeficiente de determinación), EEE (error estandar de los estimados), EMA (error medio absoluto), DW (estadístico Durbin Watson), n (tamaño de la muestra), IF (Índice de Furnival), E \% (error del modelo o sesgo), AIC (criterio de información de Akaike). Adicionalmente, se calculó el ECM (error cuadrático medio), la DA (diferencia agregada) y el PRESS (la suma de cuadrados de los residuos de los predichos); estos últimos no se muestran en los resultados para simplificar el cuadro en el cual aparecen los otros estadígrafos.

\section{Resultados}

\subsection{Modelos para estimar biomasa y carbono}

Los modelos para componentes del árbol o árbol completo, en general, presentan un ajuste muy aceptable con $\mathrm{R}^{2}$ entre 0.92 y $0.95(\mathrm{P}<0.0001)$ y errores de estimación inferiores al $8.4 \%$ (en el caso de las ramas) para la biomasa y el carbono. En hojas, el $\mathrm{R}^{2}$ fue inferior (0.87), con un error de $15.5 \%$ y $12.4 \%$ para la biomasa y el carbono, respectivamente. El árbol completo, el fuste y el componente leñoso mostraron un ajuste ligeramente superior para la biomasa y el carbono (Cuadro 1, Figura 3). En los modelos agregados para estimar biomasa y carbono por hectárea, el ajuste fue mejor $\left(\mathrm{R}^{2}>0.96 \%\right.$ ), con errores o sesgos inferiores al $0.6 \%$ (Cuadro 2, Figura 4). En las Figuras 3a y 4a, se observa el gráfico del modelo ajustado (línea central), con su banda de confianza y de predicción, así como las Figuras $3 \mathbf{b}$ y $\mathbf{4 c}$ representan el gráfico de valores predichos vs. observados.

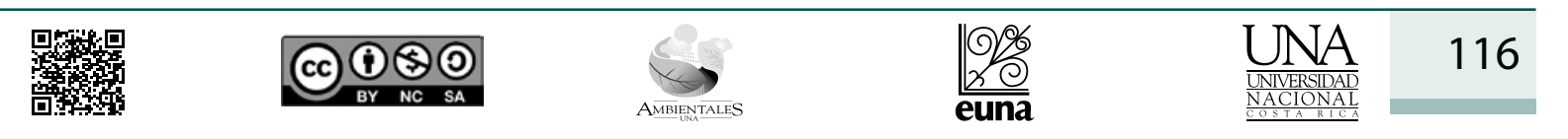




\section{Revista de CIENCIAS AMBIENTALES Tropical Journal of Environmental Sciences}

Revista de Ciencias Ambientales (Trop J Environ Sci) e-ISSN: 2215-3896

(Julio-Diciembre, 2019). Vol 53(2): 111-131 DOI: https://doi.org/10.15359/rca.53-2.5

Open Access: www.revistas.una.ac.cr/ambientales e-mail: revista.ambientales@una.ac.cr Fonseca-González W., Villalobos-Chacón R., Rojas-Vargas M.

En los modelos por árbol, a pesar del de buen ajuste, a partir de diámetros superiores a $40 \mathrm{~cm}$ muestran una tendencia a subestimar el valor de la variable por calcular (biomasa o carbono), no así para los modelos generados que usan el área basal como variable predictora.

El estadístico de Durbin-Watson se utiliza para detectar la presencia de autocorrelación en los residuos (errores de predicción) y varía entre 0 y 4 , se debe tener precaución cuando es inferior a 1. En este caso, los valores de dicho estadístico están cercanos a 2 (Cuadros 1 y 2), lo que sugiere que no hay subestimación del nivel de significación estadística.

El índice de Furnival (IF) permite comparar modelos lineales con aquellos en los cuales la variable dependiente es transformada; bajo este criterio, se elige el modelo con IF menor. El criterio de información de Akaike (AIC) mide la bondad del ajuste del modelo, es decir, describe la relación entre el sesgo y la varianza en la construcción del modelo; el modelo preferido es el que tiene menor AIC.

La correlación siempre fue positiva para la biomasa y el carbono con el diámetro; en los modelos, por árbol o por componente de este, varió entre 0.93 y 0.97 ; con área basal para los modelos agregados, en todos los casos, fue de 0.98 (Cuadros 1 y 2).

\subsection{Biomasa y carbono en la vegetación y necromasa}

En el área de estudio, para una muestra de 82 unidades con áreas basales que van desde 5 hasta $52.5 \mathrm{~m}^{2} \mathrm{ha}^{-1}$, se obtuvo un promedio de biomasa total (árboles, vegetación herbácea y necromasa) de $140.0 \mathrm{Mg} \mathrm{ha}^{-1}(\mathrm{E} \%= \pm 10.0)$, equivalente a $65.5 \mathrm{Mg} \mathrm{Cha}^{-1}(\mathrm{E} \%= \pm 10.0)$, y de 130.8 $\mathrm{Mg} \mathrm{ha}^{-1}(\mathrm{E} \%= \pm 10.5)$ de biomasa en los árboles $\left(62.3 \mathrm{Mg} \mathrm{C} \mathrm{ha}^{-1}(\mathrm{E} \%= \pm 10.5)\right.$. La biomasa entre sitios varió desde 35.3 hasta $336.3 \mathrm{Mg} \mathrm{ha}^{-1} \mathrm{y}$ de 30.3 a $334.3 \mathrm{Mg} \mathrm{ha}^{-1}$ para la biomasa total y arbórea, respectivamente. Los sitios de menor biomasa corresponden con los de menor área basal. Para el ecosistema estudiado, la necromasa y la vegetación herbácea representan solo el $6.6 \%$ de la biomasa total ( $4.9 \%$ en carbono); la raíz, el $27.7 \%$ de la biomasa total por hectárea y el $29.9 \%$ de la biomasa del árbol.

La fracción de carbono entre los componentes del árbol varió de $39.1 \%$ en hojas a $42.8 \%$ en la raíz. En la necromasa gruesa, esa fracción fue de $42.6 \%$, mientras que la vegetación herbácea y la hojarasca presentan los valores más bajos. Todos estos valores fueron estimados con un error máximo de $7.9 \%$ (Cuadro 3).

\subsection{Factores de expansión de la biomasa (FEB)}

El factor de expansión para incluir hojas, rama y raíz fue muy similar entre clases diamétricas, variando de 2.0 en la clase de $20-29.9 \mathrm{~cm}$ a 2.18 entre $30-39.9 \mathrm{~cm}$, con un promedio de 2.09 . El FEB para incluir hojas y ramas fue de 1.69 y solo para la raíz es de 1.37. En todos los casos, el FEB fue calculado con un error inferior al $1 \%$.

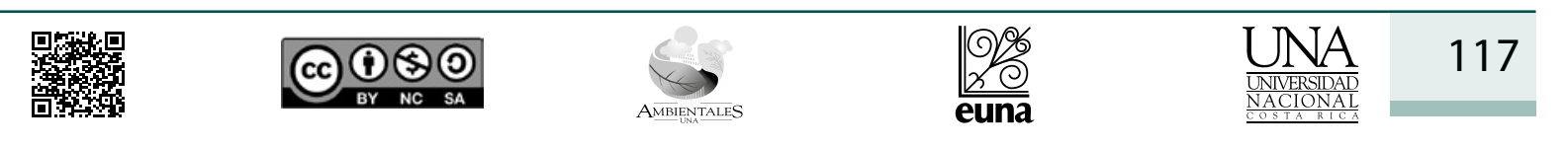




\section{Revista de CIENCIAS AMBIENTALES Tropical Journal of Environmental Sciences}

Revista de Ciencias Ambientales (Trop J Environ Sci) e-ISSN: 2215-3896

(Julio-Diciembre, 2019). Vol 53(2): 111-131 DOI: https://doi.org/10.15359/rca.53-2.5

Open Access: www.revistas.una.ac.cr/ambientales e-mail: revista.ambientales@una.ac.cr Fonseca-González W., Villalobos-Chacón R., Rojas-Vargas M.

Cuadro 1. Modelos para estimar biomasa $(\mathrm{kg})$ y carbono $(\mathrm{kg})$ para distintos componentes del árbol y para el árbol completo. Todos los modelos poseen una $\mathrm{P}<0.001$.

\begin{tabular}{|c|c|c|c|c|c|c|c|c|c|}
\hline Modelo & $\mathbf{R}$ & $\mathbf{R}^{2}$ & EEE & EMA & DW & $\mathbf{n}$ & IF & E \% & AIC \\
\hline $\begin{array}{l}\text { Btotal }=\exp (-1.66006+ \\
\left.2.26673^{\star} \ln (\text { Dap })\right)\end{array}$ & 0.97 & 0.95 & 0.384 & 0.320 & $2.149(\mathrm{P}=0.807)$ & 141 & 66.6 & 5.9 & 5.8 \\
\hline $\begin{array}{l}\text { Ctotal }=\exp (-2.40239+ \\
\left.2.26706^{*} \ln (\text { Dap })\right)\end{array}$ & 0.97 & 0.95 & 0.383 & 0.320 & $2.152(\mathrm{P}=0.812)$ & 141 & 31.7 & 5.2 & 5.1 \\
\hline $\begin{array}{l}\text { Bfuste }=\exp (-2.27978+ \\
\left.2.22575^{\star} \ln (\text { Dap })\right)\end{array}$ & 0.97 & 0.94 & 0.405 & 0.336 & $2.204(\mathrm{P}=0.888)$ & 141 & 34.5 & 6.4 & 5.2 \\
\hline $\begin{array}{l}\text { Cfuste }=\exp (-3.0139+ \\
\left.2.2258^{\star} \ln (\text { Dap })\right)\end{array}$ & 0.97 & 0.94 & 0.408 & 0.337 & $2.189(\mathrm{P}=0.869)$ & 141 & 16.7 & 5.9 & 4.4 \\
\hline $\begin{array}{l}\text { Bramas }=\exp (-3.23177+ \\
\left.2.38074^{\star} \ln (\text { Dap })\right)\end{array}$ & 0.96 & 0.93 & 0.484 & 0.409 & $2.139(\mathrm{P}=0.782)$ & 141 & 21.0 & 8.4 & 4.7 \\
\hline $\begin{array}{l}\text { Cramas }=\exp (-3.94582+ \\
\left.2.35809^{\star} \ln (\text { Dap })\right)\end{array}$ & 0.96 & 0.92 & 0.504 & 0.427 & $2.103(\mathrm{P}=0.720)$ & 141 & 10.3 & 8.4 & 4.0 \\
\hline $\begin{array}{l}\text { Braíz }=\exp (-2.96889+ \\
\left.2.1391^{\star} \ln (\text { Dap })\right)\end{array}$ & 0.96 & 0.93 & 0.409 & 0.339 & $1.856(\mathrm{P}=0.204)$ & 141 & 14.8 & 5.2 & 4.3 \\
\hline $\begin{array}{l}\text { Craíz }=\exp (-3,68729+ \\
\left.2,14055^{\star} \ln (\text { Dap }(\mathrm{cm}))\right)\end{array}$ & 0.96 & 0.93 & 0.410 & 0.340 & $1.849(\mathrm{P}=0.192)$ & 141 & 6.0 & 8.2 & 3.5 \\
\hline $\begin{array}{l}\text { Bhojas }=(1.81502+ \\
\left.0.0021515^{\star} \operatorname{Dap}^{\wedge} 2\right)^{\wedge} 2\end{array}$ & 0.93 & 0.87 & 1.130 & 0.845 & $2.246(\mathrm{P}=0.867)$ & 95 & 1.13 & 15.5 & 2.6 \\
\hline $\begin{array}{l}\text { Chojas }=(1.26774+ \\
\left.0.00135669^{\star} \operatorname{Dap}^{\wedge} 2\right)^{\wedge} 2\end{array}$ & 0.94 & 0.87 & 0.718 & 0.542 & $2.675(\mathrm{P}=0.999)$ & 95 & 0.72 & 12.4 & 1.8 \\
\hline $\begin{array}{l}\text { Bleñosa }=\exp (-1.67735+ \\
\left.2.24477^{\star} \ln (\text { Dap })\right)\end{array}$ & 0.97 & 0.94 & 0.417 & 0.345 & $2.124(\mathrm{P}=0.771)$ & 141 & 69.3 & 7.7 & 5.9 \\
\hline $\begin{array}{l}\text { Cleñoso }=\exp (-2.45315+ \\
\left.2.27001^{\star} \ln (\text { Dap })\right)\end{array}$ & 0.97 & 0.95 & 0.386 & 0.321 & $2.102(\mathrm{P}=0.724)$ & 141 & 30.8 & 6.0 & 5.1 \\
\hline
\end{tabular}

Btotal-Ctotal: biomasa-carbono del árbol completo, Bfuste-Cfuste: biomasa-carbono del fuste, Bramas-Cramas: biomasa-carbono de ramas, Braíz-Craíz: biomasa-carbono de raíz, Bhojas-Chojas: biomasa-carbono de hojas, Bleñoso-Cleñoso: biomasa-carbono de componente leñoso, exp: exponente, Dap: diámetro normal a $1.30 \mathrm{~m}$ sobre el nivel del suelo (cm), ln: logaritmo natural, R, R², EEE, EMA, DW, n, IF, E \% y AIC definidos en la metodología.
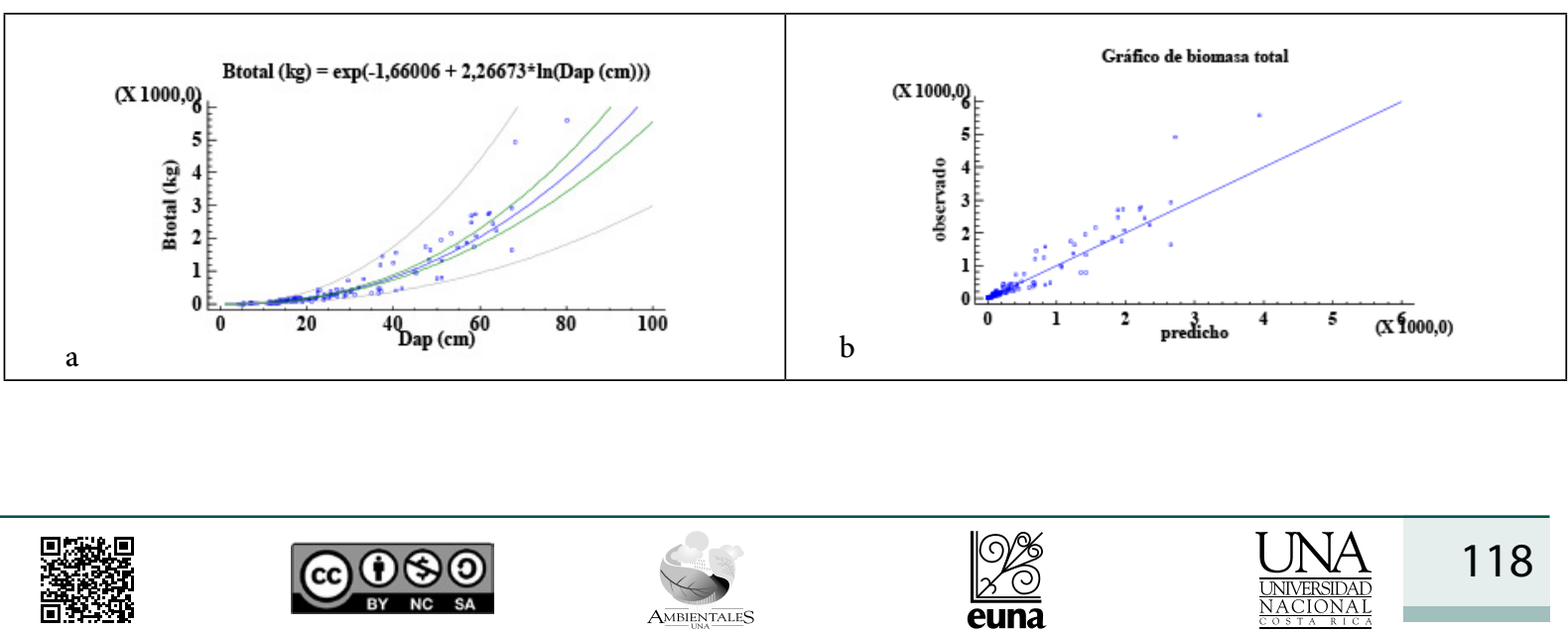
Revista de CIENCIAS AMBIENTALES
Tropical Journal of Environmental Sciences
Revista de Ciencias Ambientales (Trop J Environ Sci) e-ISSN: 2215-3896

(Julio-Diciembre, 2019). Vol 53(2): 111-131

DOI: https://doi.org/10.15359/rca.53-2.5

Open Access: www.revistas.una.ac.cr/ambientales

e-mail: revista.ambientales@una.ac.cr

Fonseca-González W., Villalobos-Chacón R., Rojas-Vargas M.

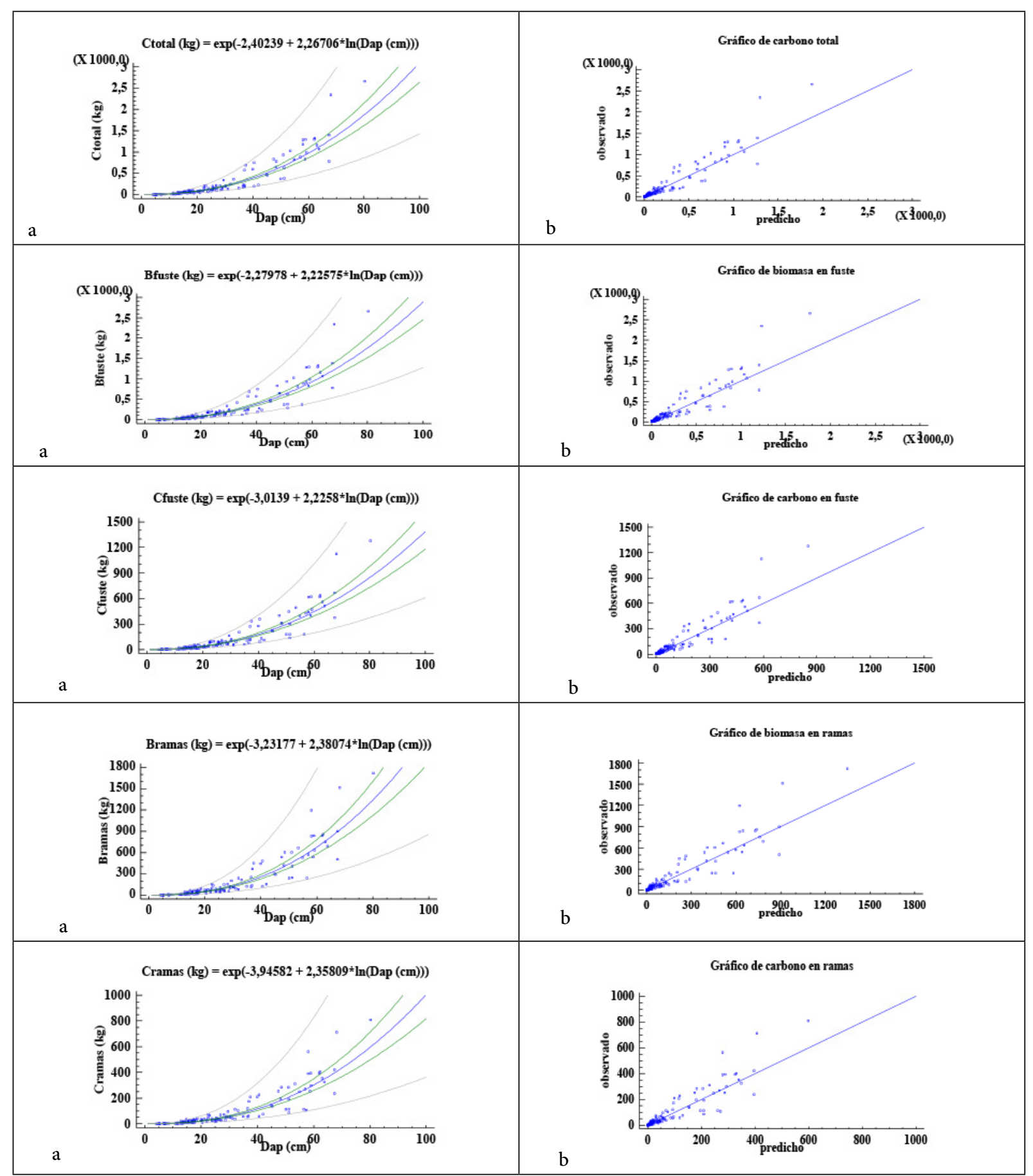

\begin{tabular}{|c|}
\hline 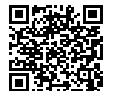 \\
\hline
\end{tabular}


Revista de CIENCIAS AMBIENTALES
Tropical Journal of Environmental Sciences
Revista de Ciencias Ambientales (Trop J Environ Sci) e-ISSN: 2215-3896

(Julio-Diciembre, 2019). Vol 53(2): 111-131

DOI: https://doi.org/10.15359/rca.53-2.5

Open Access: www.revistas.una.ac.cr/ambientales

e-mail: revista.ambientales@una.ac.cr

Fonseca-González W., Villalobos-Chacón R., Rojas-Vargas M.

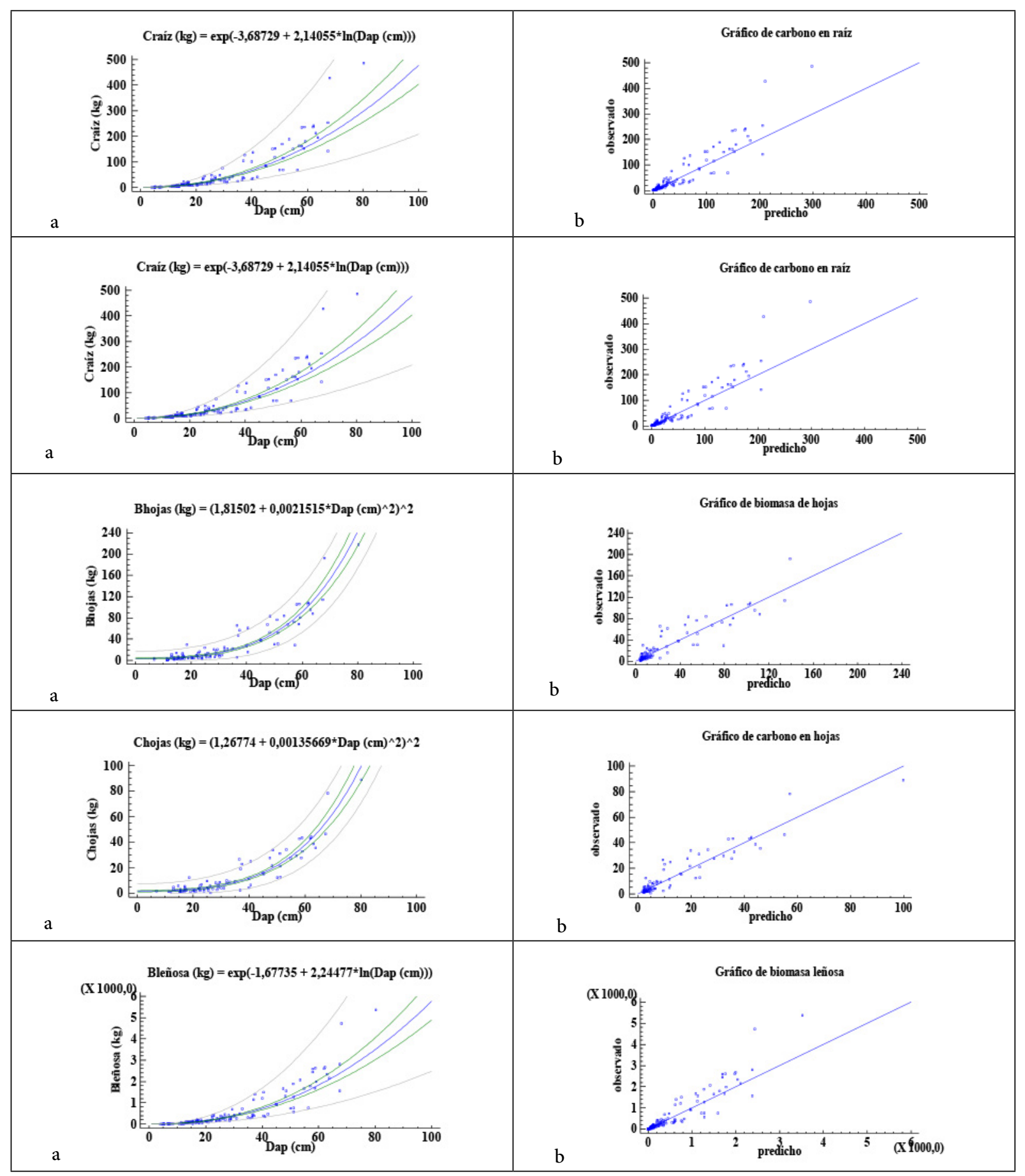




\section{Revista de CIENCIAS AMBIENTALES Tropical Journal of Environmental Sciences}

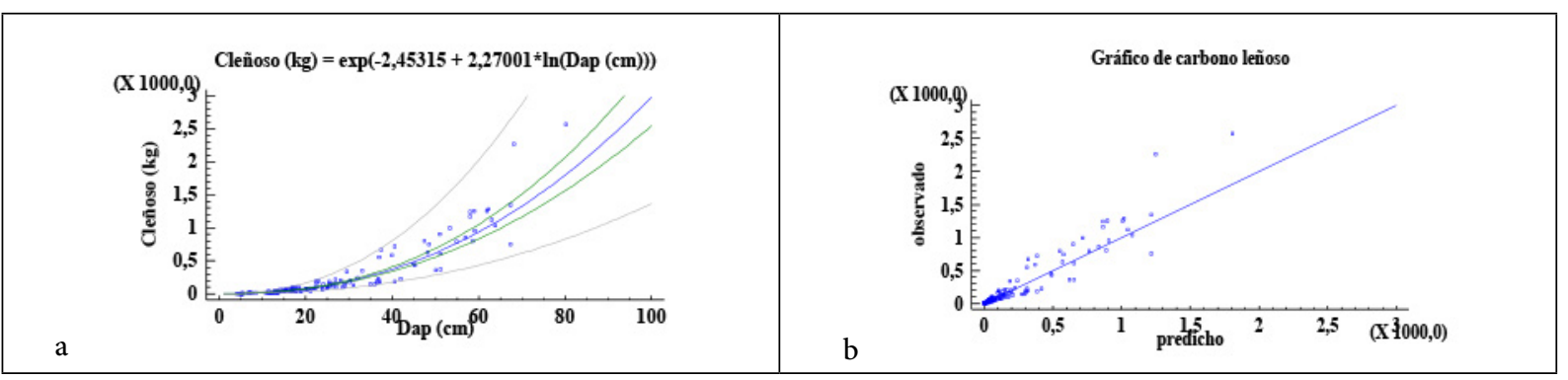

Figura 3. Modelos para estimar biomasa y carbono por componente de árbol y árbol completo, a partir del diámetro: a) el gráfico para el modelo ajustado, b) el gráfico de valores predichos vs. observados.

Cuadro 2. Modelos para estimar biomasa $\left(\mathrm{Mg} \mathrm{ha}^{-1}\right)$ y carbono $\left(\mathrm{Mg} \mathrm{ha}^{-1}\right)$. Todos los modelos poseen una $\mathrm{P}<0.001$.

\begin{tabular}{|c|c|c|c|c|c|c|c|c|c|}
\hline Modelo & $\mathbf{R}$ & $\mathbf{R}^{2}$ & EEE & EMA & DW & $\mathbf{n}$ & IF & E \% & AIC \\
\hline $\begin{array}{l}\text { Btotal }=\left(0.301575+2.46272^{\star}(G\right. \\
\wedge 0.5))^{\wedge} 2\end{array}$ & 0.98 & 0.96 & 0.537 & 0.430 & $2.215(\mathrm{P}=0.807)$ & 81 & 0.54 & 0.59 & 2.6 \\
\hline $\begin{array}{l}\log \text { Ctotal }=(1.2923+ \\
\left.0.242744^{\star} \ln (G)\right)^{\wedge} 2\end{array}$ & 0.98 & 0.96 & 0.022 & 0.018 & $2,348(\mathrm{P}=0.931$ & 81 & 3.01 & 0.33 & 1.8 \\
\hline $\begin{array}{l}\text { Barbórea }=\exp (1.78492+ \\
\left.0.99869^{\star} \ln (G)\right)\end{array}$ & 0.98 & 0.97 & 0.086 & 0.069 & $1.897(\mathrm{P}=0.281)$ & 81 & 10.9 & 0.36 & 2.49 \\
\hline $\begin{array}{l}\text { Carbóreo }=\left(-0.12897+1.71179^{\star}(G\right. \\
\wedge 0.5))^{\wedge} 2\end{array}$ & 0.98 & 0.97 & 0.330 & 0.261 & $1.854(\mathrm{P}=0.218)$ & 81 & 0.33 & 0.42 & 1.74 \\
\hline $\begin{array}{l}\text { Bleñosa }=\exp (1.72354+ \\
\left.1.00501^{\star} \ln (G)\right)\end{array}$ & 0.98 & 0.97 & 0.084 & 0.068 & $1.894(\mathrm{P}=0.276)$ & 81 & 9.49 & 0.34 & 2.44 \\
\hline $\begin{array}{l}\text { Cleñoso }=\exp (0.990397+ \\
\left.1.00429^{\star} \ln (G)\right)\end{array}$ & 0.98 & 0.97 & 0.084 & 0.068 & $1.895(\mathrm{P}=0.277)$ & 81 & 4.55 & 0.35 & 2.42 \\
\hline
\end{tabular}

Btotal-Ctotal: biomasa-carbono vegetal total (árboles, vegetación herbácea y necromasa), Barbórea-Carbóreo: biomasa-carbono de los árboles, Bleñosa- Cleñosa: biomasa-carbono de los componentes leñosos de los árboles (ramas, fuste, raíz), G: área basal ( $\left.\mathrm{m}^{2} \mathrm{ha}^{-1}\right)$, ln: logaritmo natural, exp: exponente, R, R², EEE, EMA, DW, n, ECM, DA, IF, PRESS, E\% y AIC definidos en la metodología.

Cuadro 3. Fracción de carbono por componente de biomasa

\begin{tabular}{lccccccc}
\hline Estadístico & Hojas & Ramas & Fuste & Raíz & $\begin{array}{c}\text { Necro- } \\
\text { masa gruesa }\end{array}$ & $\begin{array}{c}\text { Vegetación } \\
\text { herbácea }\end{array}$ & Hojarasca \\
\hline $\mathbf{X}$ & 39.1 & 42.7 & 42.5 & 42.8 & 42.6 & 38.5 & 33.3 \\
$\mathbf{n}$ & 46 & 30 & 31 & 37 & 26 & 18 & 27 \\
$\mathrm{~S}$ & 6.4 & 4.9 & 5.8 & 5.4 & 5.0 & 3.3 & 5.5 \\
$\mathrm{E} \%$ & 6.0 & 7.9 & 6.6 & 4.4 & 4.7 & 4.2 & 6.5 \\
\hline
\end{tabular}

X: promedio, n: tamaño de la muestra, S: desviación estándar, E \%: error relativo de muestreo.

\begin{tabular}{|c|c|c|}
\hline 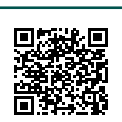 & (c) (i) () () & 121 \\
\hline
\end{tabular}


Revista de CIENCIAS AMBIENTALES
Tropical Journal of Environmental Sciences
Revista de Ciencias Ambientales (Trop J Environ Sci) e-ISSN: 2215-3896

(Julio-Diciembre, 2019). Vol 53(2): 111-131

DOI: https://doi.org/10.15359/rca.53-2.5

Open Access: www.revistas.una.ac.cr/ambientales

e-mail: revista.ambientales@una.ac.cr Fonseca-González W., Villalobos-Chacón R., Rojas-Vargas M.

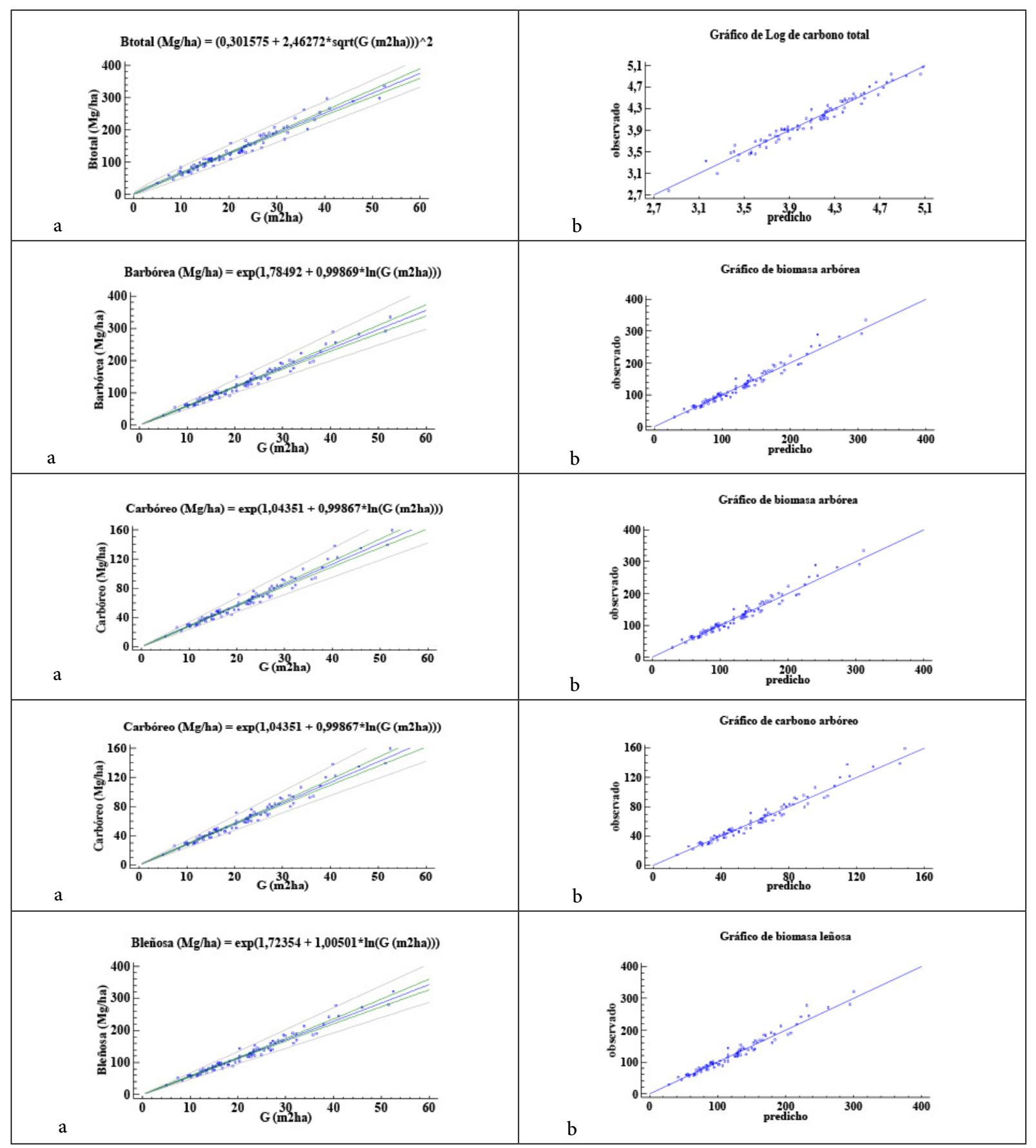
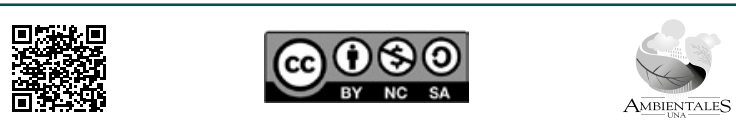


\section{Revista de CIENCIAS AMBIENTALES Tropical Journal of Environmental Sciences}

Revista de Ciencias Ambientales (Trop J Environ Sci) e-ISSN: 2215-3896

(Julio-Diciembre, 2019). Vol 53(2): 111-131 DOI: https://doi.org/10.15359/rca.53-2.5

Open Access: www.revistas.una.ac.cr/ambientales e-mail: revista.ambientales@una.ac.cr Fonseca-González W., Villalobos-Chacón R., Rojas-Vargas M.

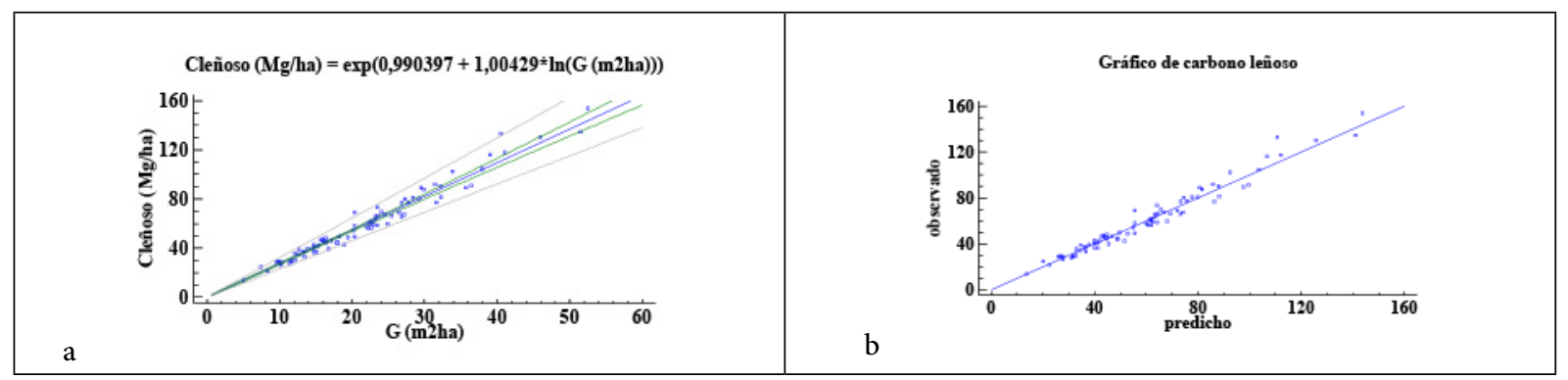

Figura 4. Modelos para estimar biomasa y carbono por hectárea, a partir del área basal: a) el gráfico para el modelo ajustado, b) el gráfico de valores predichos vs. observados.

\section{Discusión}

\subsection{Modelos para estimar biomasa y carbono}

El uso de modelos para estimar biomasa es una forma práctica y económica que determina el aporte de un ecosistema mitigar el cambio climático. Es usual que, ante la ausencia de modelos en el nivel local, se utilicen los desarrollados en regiones diferentes. Álvarez (2008) advierte sobre las limitaciones de esta práctica, ya que las condiciones ambientales (clima, suelo) y la genética influyen sobre el crecimiento y en la fijación del carbono.

Los modelos desarrollados en este trabajo para estimar biomasa y carbono por componente del árbol y para el árbol completo, en su mayoría, presentaron muy buenos ajustes $\left(\mathrm{R}^{2} \geq 0.92\right)$ en el nivel de árbol (fuste o árbol completo), con sesgos de estimación muy bajos $(\mathrm{E}<8.4 \%)$, excepto las hojas con un $\mathrm{R}^{2}$ de 0.87 y errores de estimación de 15.5 y 12.4, tanto para biomasa como para carbono, respectivamente. En los modelos agregados, el área basal mostró alta correlación con la biomasa y el carbono por hectárea, dando como resultado modelos con un ajuste muy bueno $\left(\mathrm{R}^{2} \geq 0.96\right)$, con muy bajos errores de estimación $(\mathrm{E}<0.6 \%)$. En todos los casos, el buen ajuste y bajo error demuestra mucha confiabilidad, adicionalmente, son modelos muy sencillos y prácticos porque usan como variable explicativa o predictora el diámetro o el área basal, que es muy fácil de medir o calcular y se obtiene en casi todos los inventarios.

En los modelos por árbol, el ajuste $\left(\mathrm{R}^{2} \geq 0.86\right)$ demuestra la existencia de muy buena correlación entre la biomasa o el carbono y la variable explicativa (Dap), situación similar reportada en gran variedad de estudios en ambientes similares o para ecosistemas más húmedos. En bosques secos o caducifolios, Bustos, Morales, Cambrón y Ledesma (2015) lograron un $\mathrm{R}^{2}>0.85$ para 9 especies; Martínez, Sarukhan, Pérez, Rincón, Maass, Solís y Cervantes (1992), un R² de 0.88 para bosques secos de México; mientras que Solano, Eras y Cueva (2014), en bosque seco pluviestacional en Ecuador, desarrollaron modelos con altos coeficientes de determinación que van desde 0.85 hasta 0.99 .

\section{F.




\section{Revista de CIENCIAS AMBIENTALES Tropical Journal of Environmental Sciences}

Revista de Ciencias Ambientales (Trop J Environ Sci) e-ISSN: 2215-3896

(Julio-Diciembre, 2019). Vol 53(2): 111-131 DOI: https://doi.org/10.15359/rca.53-2.5

Open Access: www.revistas.una.ac.cr/ambientales e-mail: revista.ambientales@una.ac.cr Fonseca-González W., Villalobos-Chacón R., Rojas-Vargas M.

La menor correlación entre el componente de biomasa y carbono en hojas y el diámetro podría estar afectada por la pérdida de follaje en los árboles elegidos, por tratarse de un ecosistema de bosque caducifolio, pero, además, parece existir una relación alométrica poco definida entre la biomasa de este componente, e incluso la biomasa de ramas y raíces, con variables explicativas como el diámetro y la altura que se demuestra en varios estudios. Risio (2012), en la pampa semiárida de Argentina, para Prosopis caldenia, no logró un modelo adecuado que predijera la biomasa foliar a partir del Dap y de la altura, pero el ajuste mejoró para las ramillas de $2-7 \mathrm{~cm}$ de diámetro $\left(\mathrm{R}^{2} \mathrm{aj}=0.66\right)$ y un máximo de 0.82 para el fuste. En cambio, Fonseca et al. (2009) lograron ajustes $\left(R^{2}\right)$ entre 0.89 y 0.95 para hojas y ramas, en 2 especies en plantación y entre 0.83 y 0.91 para un grupo de 35 especies de mayor IVI en bosque natural. También, Jiménez, Fonseca y Pazmiño (2019) alcanzaron un $\mathrm{R}^{2}$ de 0.96 para dichos componentes con Guazuma ulmofolia, mientras que, en estos 2 últimos estudios, el $\mathrm{R}^{2}$ para el fuste y el árbol completo superó el 0.93 . Los modelos agregados para estimar biomasa o carbono por hectárea son una opción más práctica que cuantifica el aporte del bosque a mitigar el cambio climático, porque evita hacer cálculos individuales para árboles o agrupaciones (clases diamétricas), y luego llevar a cabo las extrapolaciones por hectárea, pasos en los cuales se cometen errores, si no se tiene cuidado y experiencia. Este tipo de modelos es poco frecuente en la literatura, pero presenta muy buenos ajustes, como los logrados por Fonseca, Rey-Benayas y Alice (2011); Fonseca, Alice y Rey-Benayas (2012), y Martínez et al. (1992), similares a los obtenidos en este trabajo $\left(R^{2} \geq 0.96\right)$.

\subsection{Biomasa y carbono en la vegetación y necromasa}

La variabilidad observada en la acumulación de biomasa y carbono, producto de las diferencias en área basal entre sitios (implica mayor o menor cobertura vegetal), puede deberse a la edad del ecosistema y a la calidad de sitio (productividad del sitio), resultado de disturbios (incendios, manejo, degradación del bosque, etc.); estos últimos elementos son muy repetitivos en la zona (Álvarez et al., 2012; Miles et al., 2006). Cualquiera que sea el motivo, la biomasa presenta la particularidad de ser una variable altamente heterogénea (Segura y Kanninen, 2002).

Para el ecosistema estudiado, la necromasa y la vegetación herbácea representan solo el 6.6 $\%$ de la biomasa total (4.9 \% en carbono), cifra por considerar para valorar si en futuros estudios es conveniente evaluar estos componentes, dada la inversión en tiempo que conlleva. Este comportamiento ocurre para otros sitios, por ejemplo, Torres et al. (2012) mencionan que las especies leñosas representan casi el $100 \%$.

El valor de carbono $\left(65,5 \mathrm{Mg} \mathrm{C}^{-1}\right.$ en la biomasa total y $62,3 \mathrm{MgC} \mathrm{ha}^{-1}$ en la biomasa de los árboles) es congruente con lo reportado por Houghton y Skole (1990), quienes encontraron que el bosque seco tropical tiene almacenado un promedio de $65 \mathrm{MgC} \mathrm{ha}^{-1}$, con incrementos de 6.2 Mg C ha ${ }^{-1} a_{n} \mathrm{O}^{-1}$; Zhaohua, Birdsey, Andrade y Johnson (2014), por su parte, calcularon una media de 56,6 $\mathrm{Mg} \mathrm{C}^{-1}{ }^{-1}$ con una variabilidad que osciló entre 5.0 y $115.0 \mathrm{Mg} \mathrm{C}^{-1}$. El reporte de Torres et al. (2012) es inferior, con una biomasa total de $84.6 \mathrm{Mg} \mathrm{ha}^{-1}\left(42.3 \mathrm{Mg} \mathrm{C}^{-1}\right.$ ) y 88.0 $\mathrm{Mg} \mathrm{ha}^{-1}$ (44.0 Mg C ha $\left.{ }^{-1}\right) 2$ años después. Para regiones áridas de Colombia, Fernández, Melo,

\begin{tabular}{|c|c|c|c|c|c|}
\hline 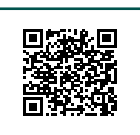 & (c) (i) @) (2) & $\underset{\text { AMADENTILIS }}{S}$ & $\frac{126}{20}$ & 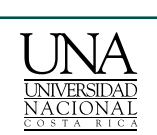 & 124 \\
\hline
\end{tabular}




\section{Revista de CIENCIAS AMBIENTALES Tropical Journal of Environmental Sciences}

Revista de Ciencias Ambientales (Trop J Environ Sci) e-ISSN: 2215-3896

(Julio-Diciembre, 2019). Vol 53(2): 111-131 DOI: https://doi.org/10.15359/rca.53-2.5

Open Access: www.revistas.una.ac.cr/ambientales e-mail: revista.ambientales@una.ac.cr Fonseca-González W., Villalobos-Chacón R., Rojas-Vargas M.

Cogollo, Álvarez y Adarve (2011) reportan una biomasa aérea promedio de $55 \pm 31 \mathrm{Mg} \mathrm{ha}^{-1}$, con un rango de 8-141 $\mathrm{Mg} \mathrm{ha}^{-1}$, así como Montes, Parrado y Álvarez (2017) calcularon entre 33.52 y 46.07 Mg C ha-1 con una media de 42.54 $\mathrm{Mg} \mathrm{ha}^{-1}, 24.05 \mathrm{Mg} \mathrm{ha}^{-1}$; mientras tanto, Martínez, Alanís, Jiménez, Yerena, y Gómez (2015), para un matorral espinoso tamaulipeco, cuantificaron 21.2 $\mathrm{Mg} \mathrm{ha}^{-1}$ de biomasa arbórea aérea.

Un caso extremo de biomasa total en individuos con Dap $\geq 10 \mathrm{~cm}$ en bosques secos semideciduos de Bolivia es de $260 \mathrm{Mg} \mathrm{ha}^{-1}$ de biomasa y $125 \mathrm{Mg} \mathrm{ha}^{-1}$ de carbono (Araujo, Arroyo, Killeen y Saldias, 2006). Otros valores de biomasa y carbono para ecosistemas secos son reportados por Martínez et al. (1992).

Las similitudes o diferencias entre los valores de biomasa o carbono calculados en este estudio con los desarrollados en otras latitudes en ecosistemas caducifolios pueden deberse a condiciones ambientales, actividades antrópicas y diferencias metodológicas (componentes de biomasa medidos, diámetro mínimo considerado, especies evaluadas y método usado para determinar tanto la biomasa como el carbono).

La biomasa (carbono) presente en la raíz es un componente poco estudiado por el grado de dificultad que conlleva la evaluación (Dixon, 1995; Fonseca, 2017) y, para el caso de ecosistemas áridos o semiáridos, cobra más importancia por el mayor aporte a la biomasa total (Dixon, 1995).

En esta investigación, la raíz aportó el 27.7 \% de la biomasa vegetal por hectárea y el $29.9 \%$ de la biomasa del árbol, similar a lo indicado por Torres et al. (2012) (22,5\%), más bajo que lo citado por Sierra, del Valle y Orrego (2001) (72 \%). El mayor aporte de la biomasa radical se da en estadios sucesionales tempranos (Sierra et al., 2001).

Para estimar el carbono almacenado en la biomasa, existen 2 formas, la primera (determinación en laboratorio) es más laboriosa y con un costo económico alto, porque requiere recolección de muestras en el campo y su posterior procesamiento. La segunda opción no necesita ninguna inversión y consiste en asumir un valor de 0.47 (conocido como fracción de carbono) para convertir la biomasa seca en carbono (IPCC, 2006). En este trabajo, la facción de carbono para todos los componentes de biomasa (entre 33.3 y $42.8 \%$ ) es inferior a la aceptada por el IPCC (2006) y a la usada en muchos estudios. Tal diferencia es tan marcada que supera un $15 \%$ a todos los componentes (ramas-fuste-raíz $15 \%$, hojas $21.8 \%$, vegetación herbácea $23 \%$ y necromasa $33.4 \%$ ). Valores similares a los encontrados en el presente estudio, los cuales demuestran que la fracción o el contenido de carbono varía con la especie y el tejido o componente, fueron también reportados por Castaño y Bravo (2012) y Fonseca et al. $(2011,2012)$.

\subsection{Factores de expansión de la biomasa (FEB)}

Su cálculo se fundamenta en la necesidad de estimar la biomasa de hojas, ramas y raíces, ya que, en los inventarios, generalmente, se estima solo la biomasa del fuste. En este estudio, el FEB para el incluir la biomasa de hojas, ramas y raíces fue de 2.04, de 1.69 para hojas y ramas y de 1.37 solo para la raíz; esto quiere decir que por un megagramo $(\mathrm{Mg})$ de biomasa de fuste existe $1.04 \mathrm{Mg}$ entre hojas, ramas y raíz o $0.37 \mathrm{Mg}$ de raíz.

\begin{tabular}{|c|c|c|}
\hline 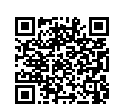 & (c) (i) (-) & 125 \\
\hline
\end{tabular}




\section{Revista de CIENCIAS AMBIENTALES Tropical Journal of Environmental Sciences}

Revista de Ciencias Ambientales (Trop J Environ Sci) e-ISSN: 2215-3896

(Julio-Diciembre, 2019). Vol 53(2): 111-131 DOI: https://doi.org/10.15359/rca.53-2.5

Open Access: www.revistas.una.ac.cr/ambientales e-mail: revista.ambientales@una.ac.cr Fonseca-González W., Villalobos-Chacón R., Rojas-Vargas M.

En general, los FEB reportados en la literatura se han calculado solo para incluir el follaje y las ramas; para esto, el IPCC (2006) y Dixon (1995) recomiendan una relación de 1.6, muy similar a lo calculado en este trabajo (1.69). Según Álvarez (2008), el factor de expansión de biomasa para ecosistemas secos se encuentra entre 1.9 y 2.4; Solano et al. (2014) calculó cifras similares para algunas especies de bosque seco pluviestacional en Ecuador (1.72 para Cordia alliodora, 1.41 para Eriotheca ruizii y 1.75 para Tabebuia chrysantha).

El FEB (1.69) del ecosistema estudiado está en el rango reportado para otras especies en bosque natural o plantadas en ambientes más húmedos; por ejemplo, 1.6 promedio para varias especies en bosque natural (Segura et al., 2000), 1.44 para un grupo de 35 especies en bosque secundario (Fonseca et al., 2009) y 1.37 para Pinus pseudostrobus (Domínguez, Aguirre, Jiménez, Rodríguez y Díaz, 2009).

El factor de expansión para raíz (1.37) es superior a lo reportado por Cairns (1997) (1.25 con especies deciduas, independientemente de la latitud o la textura edáfica en la que se desarrollen los árboles), y Fonseca et al. (2009) (1.25 para 35 especies en bosques secundarios y 1.3 para 2 especies en plantación). Ese factor, igualmente, es inferior al calculado por Gaston, Brown, Lorenzini y Singhi (1998) para bosques y sabanas en ambientes áridos y semiáridos (1.47), así como para varias especies deciduas de la península ibérica (Ruíz, Rio y Montero, 2011).

\section{Conclusiones}

Los modelos elegidos con el fin de estimar la biomasa o el carbono para los diferentes componentes del árbol, el árbol completo, así como los modelos agregados presentaron una bondad de ajuste muy buena $\left(\mathrm{R}^{2}>0.87\right)$ con bajos errores de estimación. Asimismo, muestran la ventaja de la simplicidad en su uso, por utilizar como variable predictora el diámetro o el área basal, ambos fáciles de medir o calcular, por lo cual se convierten en una herramienta muy útil para estimar la biomasa o el carbono, con precisión y confiabilidad; esto, si son empleados en ecosistemas similares y para el rango de distribución diamétrica o de área basal con los que fueron construidos.

La biomasa y el carbono determinados en el área estudiada, tanto en el nivel de ecosistema como para la masa arbolada, así como los valores de fracción de carbono en la biomasa y los factores de expansión, están entre los rangos citados en otras investigaciones. De tal forma, la información generada se convierte en indicadores nacionales que permiten y facilitan calcular, con mayor exactitud, el aporte del ecosistema a la mitigación del cambio climático.

\section{Agradecimientos}

Este proyecto fue financiado por el aporte de la Universidad Nacional de Costa Rica y de la Agencia para la Cooperación Alemana (GIZ), por la contribución del Sistema Nacional de Áreas de Conservación de Costa Rica (SINAC) y por el apoyo de los propietarios de los terrenos,

(cc)




\section{Revista de CIENCIAS AMBIENTALES Tropical Journal of Environmental Sciences}

Revista de Ciencias Ambientales (Trop J Environ Sci) e-ISSN: 2215-3896

(Julio-Diciembre, 2019). Vol 53(2): 111-131 DOI: https://doi.org/10.15359/rca.53-2.5

Open Access: www.revistas.una.ac.cr/ambientales e-mail: revista.ambientales@una.ac.cr Fonseca-González W., Villalobos-Chacón R., Rojas-Vargas M.

quienes permitieron la realización del estudio. Al igual que a dichos actores, se agradece a la Revista y las personas revisoras anónimas por sus observaciones.

\section{Referencias}

Álvarez, E. y Marín, M. A. (2011). Manual de procedimientos analíticos para suelos y plantas. México: Universidad Autónoma de Chapingo.

Álvarez, G. (2008). Modelos alométricos para la estimación de biomasa aérea de dos especies nativas en plantaciones forestales del trópico de Cochabamba, Bolivia. (Tesis de Maestría). Centro Agronómico Tropical de Investigación y Enseñanza (CATIE), Turrialba, Costa Rica.

Álvarez, E., Mendoza, I., Pacheco, M., Restrepo, Z., Benítez, D., Gutiérrez, T. D., ... y Gómez, F. (2012). ¿Por qué implementar estudios de largo plazo en el Bosque Seco del Caribe colombiano? Revista Intrópica, 7, 97-113. ISSN 1794-161X

Araujo, A., Arroyo, L., Killeen, T. J. \& Saldias, M. (2006). Dinámica del bosque, incorporación y almacenamiento de biomasa y carbono en el Parque Nacional Noel Kempff Mercado. Ecología en Bolivia, 41(1), 24-45.

Bustos, D., Morales, A., Cambrón, V. H. y Ledesma, M. (2015). Estimación de la capacidad de captura de carbono de la vegetación del parque nacional El Cimatario, Querétaro. Digital Ciencia@uaqro,1-11.INCIA@UAQRODIGITALCIENCIA@UAQRO

Cairns, M., Brown, S., Helmer, E. H. y Baumgardner, G. (1997). Root biomass allocation in the world's upland forests. Oecología, 111(1), 1-11. https://doi.org/10.1007/s004420050201

Castaño, J. y Bravo, F. (2012). Variation in carbon concentration and basic density along stems of sessile oak (Quercus petraea (Matt.) Liebl.) and Pyrenean oak (Quercus pyrenaicaWilld.) in the Cantabrian Range (NW Spain). Annals of Forest Science, 69(6), 633-672. http:// doi. org/10.1007/s13595-012-0183-6

Chave, J., Réjou-Méchain, M., Búrquez, A., Chidumayo, E., Colgan, M., Delitti, W., ... y Vieilledent, G. (2014). Improved pantropical allometric models to estimate the above ground biomass of tropical forests. Global Change Biology, 20, 3177-319. https://doi.org/10.1111/ gcb.12629

Cifuentes, M., Henry, M., Méchain, M., López, O., Wayson, C., Michel, J. M., ... y Westfal, J. (2015). Overcoming obstacles to sharing data on tree allometric equations. Annals of Forest Science, 72(6), 789-794. https://doi.org/10.1007/s13595-015-0467-8

Dixon, R. (1995). Agroforestry systems: sources or sinks of greenhouse gases? Agroforestry systems, 31, 99-116. https://doi.org/10.1007/BF00711719

\begin{tabular}{|c|c|c|}
\hline 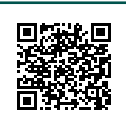 & (cc) (i) & 127 \\
\hline
\end{tabular}




\section{Revista de CIENCIAS AMBIENTALES Tropical Journal of Environmental Sciences}

Revista de Ciencias Ambientales (Trop J Environ Sci) e-ISSN: 2215-3896

(Julio-Diciembre, 2019). Vol 53(2): 111-131 DOI: https://doi.org/10.15359/rca.53-2.5

Open Access: www.revistas.una.ac.cr/ambientales e-mail: revista.ambientales@una.ac.cr Fonseca-González W., Villalobos-Chacón R., Rojas-Vargas M.

Domínguez, G., Aguirre, O., Jiménez, J., Rodríguez, R. y Díaz, J. (2009). Biomasa aérea y factores de expansión de especies arbóreas en bosques del sur de Nuevo León. Revista Chapingo Serie Ciencias Forestales y del Ambiente, 15(1), 59-64.

Fernández, F., Melo, O., Cogollo, A., Álvarez, E. y Adarve, J. (2011). Almacenamiento de carbono del bosque seco del Caribe y otras regiones de Colombia. En J. F. Quintero y S. M. Estrada (eds.), Memorias I Encuentro Regional de Investigadores en Ciencias de la Vida - Sinergia Caribe 31 de octubre al 4 de noviembre de 2011. Santa Marta: Editorial de la Universidad del Magdalena.

Fonseca, W. (2017). Revisión de métodos para el monitoreo de biomasa y carbono vegetal en ecosistemas forestales tropicales. Revista de Ciencias Ambientales (Trop. J. Environ. Sci.), 51(2), 91-109. http://dx.doi.org/10.15359/rca.51-2.5

Fonseca, W., Alice, F. y Rey-Benayas, J. M. (2009). Modelos para estimar la biomasa de especies nativas en plantaciones y bosques secundarios en la zona Caribe de Costa Rica. Bosque, 30(1), 36-47. https://doi.org/10.4067/s0717-92002009000100006

Fonseca, W., Alice, F. y Rey-Benayas, J. M. (2011b). Carbon accumulation in the biomass and soil of different aged secondary forests in the humid tropics of Costa Rica. Forest Ecology and Management, 262, 1400-1408. https://doi.org/10.1016/j.foreco.2011.06.036

Fonseca, W., Alice, F. y Rey-Benayas, J. M. (2012). Carbon accumulation in aboveground and belowground biomass and soil of different age native forest plantations in the humid tropical lowlands of Costa Rica. New Forests, 43(2), 197-211. https://doi.org/10.1007/ s11056-011-9273-9

Gaston, G., Brown, S., Lorenzini, M. y Singhi, K. (1998). State and change in carbon pools in the forests of tropical Africa. Global Change Biology, 4, 97-114. https://doi. org/10.1046/j.1365-2486.1998.00114.x

Houghton, R. A. y Skole, D. L. (1990). Carbon. En B. L. Turner, W. C. Clark, R. W. Kates, J. F. Richards, J. T. Mathews y. B. Meyer (eds.), The Earth as Transformed by Human Action (pp. 393-408). Cambridge: Cambridge University Press.

Intergovernmental Panel of Climate Change. (2006). Directrices del IPCC de 2006 para los inventarios nacionales de gases de efecto invernadero. Agricultura, silvicultura y otros usos de la tierra (volumen 4). Disponible en https://www.ipcc-nggip.iges.or.jp/public/2006gl/spanish/ pdf/4_Volume4/V4_04_Ch4_Forest_Land.pdf

Intergovernmental Panel of Climate Change. (2013). Cambio climático 2013: bases físicas. Contribución del Grupo de trabajo I al Quinto Informe de Evaluación del Grupo Intergubernamental de Expertos sobre el Cambio Climático. Resumen para responsables de políticas. Disponible en www.climatechange2013.org

\begin{tabular}{|c|c|c|}
\hline 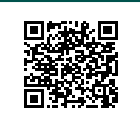 & (c) & 128 \\
\hline
\end{tabular}




\section{Revista de CIENCIAS AMBIENTALES Tropical Journal of Environmental Sciences}

Revista de Ciencias Ambientales (Trop J Environ Sci) e-ISSN: 2215-3896

(Julio-Diciembre, 2019). Vol 53(2): 111-131 DOI: https://doi.org/10.15359/rca.53-2.5

Open Access: www.revistas.una.ac.cr/ambientales e-mail: revista.ambientales@una.ac.cr Fonseca-González W., Villalobos-Chacón R., Rojas-Vargas M.

Jiménez, E., Fonseca, W y Pazmiño, L. (2019). Sistemas silvopastoriles y cambio climático: estimación y predicción de biomasa arbórea. La Granja: Revista de Ciencias de la Vida, 29(1), 45-55. http://doi.org/10.17163/lgr.n29.2019.04

Le Quéré, C., Andres, R. J., Boden, T., Conway, T., Houghton, R. A., House, J. I., ... y Zeng, N. (2012). The global carbon budget 1959-2011. Earth System Science Data Discussions, 5, 1107 1157. https://doi.org/10.5194/essdd-5-1107-2012

Martínez, A., Sarukhan, J., Pérez, J., Rincón, E., Maass, J. M., Solís, A. y Cervantes, L. (1992). Above-ground phytomass of a tropical deciduous forest on the coast of Jalisco, México. Journal of Tropical Ecology, 8, 87-96. Disponible en http://www.jstor.org/stable/2559815.

Martínez, D., Alanís, E., Jiménez, J., Yerena, J. y Gómez, J. (2015). Contenido de carbono en un sistema agroforestal pastorilsilvícola del matorral espinoso tamaulipeco. Ciencia UANL, 18(74),58-62. Recuperado de: http://cienciauanl.uanl.mx/?p=4686

Miles, L., Newton, A. C., De Fries, R. S., Ravilious, C., May, I., Blyth, S., .. y Gordon, J. E. (2006). A global overview of the conservation status of tropical dry forests. Journal of Biogeography, 33(3), 491-505. https://doi.org/10.1111/j.1365-2699.2005.01424.x

Montes, C., Parrado, A. y Álvarez. E. (2017). Tipos funcionales de plantas como estimadores de carbono en bosque seco del Caribe colombiano. Revista Mexicana de Biodiversidad, 88, 241 249. https://doi.org/10.1016/j.rmb.2017.01.006

Phillips, O. L., Aragão, L. E., Lewis, S. L., Fisher, J. B., Lloyd, J., López, G., ... y Torres, A. (2009). Drought Sensitivity of the Amazon Rainforest. Science, 323(5919), 1344-1347. http://doi. org/10.1126/science.1164033

Phillips, O. L., van der Heijden, G., Lewis, S. L., López, G., Aragão, L. E., Lloyd, J., ... y Vilanova, E. (2010). Drought mortality relationships for tropical forests. New Phytologist, 187, 631-646. http://doi.org/10.1111/j.1469-8137.2010.03359.x.

Risio, L. (2012). Cuantificación de biomasa y carbono en bosques nativos de Prosopis caldenia (Burkart) en la Pampa semiárida, Argentina. Valladolid, España: Universidad de Valladolid, Campus de Palencia, Escuela Técnica Superior de Ingenierías Agrarias.

Ruíz, R., Rio, M. y Montero, G. (2011). New models for estimating the carbon sink capacity of Spanish softwood. Species. Forest systems, 20(1), 176-188. https://doi.org/10.5424/ fs/2011201-11643

Salas, C. (2002). Ajuste y validación de ecuaciones de volumen para un relicto del bosque de Roble-Laurel-Lingue. Bosque, 23(2), 81-92. http://dx.doi.org/10.4067/ S0717-92002002000200009

\begin{tabular}{|c|c|c|}
\hline 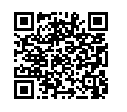 & (c) & 129 \\
\hline
\end{tabular}




\section{Revista de CIENCIAS AMBIENTALES Tropical Journal of Environmental Sciences}

Revista de Ciencias Ambientales (Trop J Environ Sci) e-ISSN: 2215-3896

(Julio-Diciembre, 2019). Vol 53(2): 111-131 DOI: https://doi.org/10.15359/rca.53-2.5

Open Access: www.revistas.una.ac.cr/ambientales e-mail: revista.ambientales@una.ac.cr Fonseca-González W., Villalobos-Chacón R., Rojas-Vargas M.

Segura, M. y Kanninen, M. (2002). Inventario para estimar carbono en ecosistemas forestales. En L. Orozco y C. Brumer (eds.), Inventarios forestales para bosques latifoliados en América Central (pp. 173-212) (Serie Técnica, Manual Técnico N. ${ }^{\circ}$ 50). Turrilaba, Costa Rica: CATIE.

Segura, M., Kanninen, M., Alfaro, A. y Campos, J. J. (2000). Almacenamiento y fijación de carbono en bosques de bajura de la zona atlántica de Costa Rica. Revista Forestal Centroamericana, 30, 23-28.

Segura, M. y Andrade, H. (2008). ¿Cómo hacerlo? ¿Cómo construir modelos alométricos de volumen, biomasa o carbono de especies leñosas perennes? Agroforestería de las Américas, $46,89-96$.

Sierra, C. A., del Valle, J. I. y Orrego, S. A. (2001). Ecuaciones de biomasa de raíces y sus tasas de acumulación en bosques sucesionales y maduros tropicales de Colombia. Simposio internacional medición y monitoreo de la captura de carbono en ecosistemas forestales, del 18 al 20 de octubre. Valdivia, Chile.

Solano, D., Eras, V. y Cueva, K. (2014). Generación de modelos alométricos para determinar biomasa aérea a nivel de especies, mediante el método destructivo de baja intensidad para el estrato de bosque seco pluviestacional del Ecuador. Revista Cedamaz, 4(1), 32-44.

Torres, A. M., Adarve, J. B., Cárdenas, M., Vargas, J. A., Londoño, V., Rivera, K., ... y González, A. M. (2012). Dinámica sucesional de un fragmento de bosque seco tropical del Valle del Cauca, Colombia. Revista Biota Colombiana, 13(2), 66-85.

Vieira, D. y Scariot, A. (2011). Principles of Natural Regeneration of Tropical Dry Forests for Restoration. Restoration Ecology, 14(1), 11-20. https://doi.org/10.1111/j.1526-100X.2006.00100.x

Zhaohua, D., Birdsey, R. A., Andrade J. L. y Johnson, K. D. (2014). Evaluación de la dinámica del carbono en el bosque tropical semideciduo de la península de Yucatán. Montreal, Canadá: Comisión para la Cooperación Ambiental de América del Norte.

\begin{tabular}{|c|c|c|c|c|c|}
\hline 唃要 & (c) (i) (5) & $\underset{\text { AMBENTALES }}{\leftrightarrow}$ & $\frac{9 \%}{2 \%}$ & 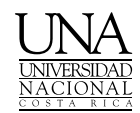 & 130 \\
\hline
\end{tabular}




\section{Revista de CIENCIAS AMBIENTALES Tropical Journal of Environmental Sciences}

Revista de Ciencias Ambientales (Trop J Environ Sci)

e-ISSN: 2215-3896

(Julio-Diciembre, 2019). Vol 53(2): 111-131

DOI: https://doi.org/10.15359/rca.53-2.5

Open Access: www.revistas.una.ac.cr/ambientales

e-mail: revista.ambientales@una.ac.cr Fonseca-González W., Villalobos-Chacón R., Rojas-Vargas M.

\section{Apéndices}

Apéndice 1. Especies usadas para generar los modelos de biomasa y carbono

\begin{tabular}{|c|c|c|c|}
\hline Especie & Familia & Especie & Familia \\
\hline Albizia niopoides & Fabacea mimosoidea & Hymenaea courbaril & Fabaceae caesalpinioidea \\
\hline Allophylus occidentalis & Sapindaceae & Lonchocarpus minimiflorus & Fabaceae papilionoidea \\
\hline Apeiba tibourbou & Tiliaceae & Lonchocarpus sp. & Fabaceae papilionoidea \\
\hline Bravaisia integerrima & Acanthaceae & Luhea candida & Tiliaceae \\
\hline Bursera simaruba & Simaroubaceae & Plumeria rubra & Apocynaceae \\
\hline Byrsonima crassifolia & Malpighiaceae & Punica granatum & Punicaceae \\
\hline Calycophyllum candidissimum & Rubiaceae & Quercus oleoides & Fagaceae \\
\hline Casearia aculeata & Flacourtiaceae & Randia sp. & Rubiaceae \\
\hline Cedrela odorata & Meliaceae & Rehdera trinervis & Verbenaceae \\
\hline Chomelia spinosa & Rubiaceae & Rollinea sp. & Annonaceae \\
\hline Cochlospermum vitifolium & Bixaceae & Samanea saman & Fabaceae caesalpinioidea \\
\hline Cordia alliodora & Boraginaceae & Scianodendrum excelsum & Araliaceae \\
\hline Cordia gerascanthus & Boraginaceae & Semialarium mexicanum & Hippocrateaceae \\
\hline Cupania guatemalensis & Sapindaceae & Simarouba glauca & Simaroubaceae \\
\hline Diospyros salicifolia & Ebenaceae & Spondias mombin & Anacardiaceae \\
\hline Enterolobium cyclocarpum & Fabacea mimosoidea & Spondias purpurea & Anacardiaceae \\
\hline Eugenia xalapensis & Myrtaceae & Swietenia macrophylla & Meliaceae \\
\hline Genipa americana & Rubiaceae & Tabebuia impetiginosa & Bignoniaceae \\
\hline Gliricidia sepium & Fabacea papilionoidea & Tabebuia ochracea & Bignoniaceae \\
\hline Godmania aesculifolia & Bignoniaceae & Thouinidium decandrum & Sapindaceae \\
\hline Guazuma ulmifolia & Sterculiaceae & Trichillia martiana & Meliaceae \\
\hline
\end{tabular}

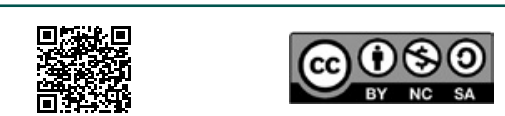

